\title{
Syndrome of downward gaze paralysis, amnesia and hypersomnolence
}

\author{
N. Nagaratnam, D.F. Ghougassian and V. Mugridge \\ Geriatric and Rehabilitation Unit, Blacktown Hospital, Blacktown, NSW 2148, Australia
}

\begin{abstract}
Summary: A 60 year old woman with computed tomographic-verified bilateral thalamic infarction is described. She demonstrated downward gaze paralysis, amnesia and hypersomnolence. On the functional level such tasks as walking and eating were disconcerting. Although there have been only a few reports of the complete triad it may be more common than realized and represents a distinct syndrome that can be identified by the bedside.
\end{abstract}

\section{Introduction}

Reports of cases with the complete clinical triad of downward gaze paralysis, amnesia and hypersomnolence are few; ${ }^{1}$ it constitutes a lacunar syndrome. ${ }^{2}$ It is essential to delineate the different type of patients with lacunes so as to outline appropriate management procedures. $^{3}$

\section{Case report}

A 60 year old widow was effectively taking care of her cooking and shopping till the day she was hospitalized with central chest pain. She had suffered an acute myocardial infarction with lateral wall ischaemia and enzyme rise. Her condition remained stable. While in hospital she was noticed to be confused and drowsy at times. She was seen by a neurologist one month after admission who felt that she had multi-infarct dementia. An electroencephalogram showed increased slow wave activity which was non-specific. The computed tomographic (CT) scan showed the ventricles normal in position and size. A low attenuation area was seen at the caudal aspect of the left basal ganglia and was thought to be the upper part of the suprasellar cistern.

There was a history of long-standing hypertension, two cerebro-vascular accidents, 6 and 4 years earlier, and non-insulin dependent diabetes.

One of her daughters had noticed deterioration in her memory and difficulty in walking. She could not focus her eyes on her food and this interfered with her

Correspondence: N. Nagaratnam, F.R.A.C.P., F.R.C.P.A. Accepted: 15 June 1989 eating. She was extremely drowsy during the da There was some uncertainty just when all these arose

Neurological examination found the patient to bs somewhat sluggish with reduced attention span, laps ing to apparent sleep when not actively spoken to $\mathbb{\Phi}$ Verbal comprehension was normal for simple cons $\vec{\varphi}$ mands but was impaired for the more complex. She was orientated only to person and was unable to do simple arithmetic. Her recent and remote memoriess were markedly impaired. There was no confabulation. There was paralysis of conjugate downward gaze. The other external ocular movements were normal except for minimal diminution in convergence. The pupillary reflexes were normal. There was no weakness in her limbs but the reflexes were brisk in all her limbs with bilateral extensor plantar responses. She had mild diminution of vibration sensibility in both legs. Her gait was unsteady with a tendency to list to one side and she walked with her head thrust forwards. There were no cerebellar signs.

The carotids were palpable with no audible bruits. There was no evidence of cardiac decompensation and the lungs were clear. The peripheral pulses were poorly felt. The biochemical tests including vitamin B12 and folic acid levels were normal. A repeat CT scan 6 weeks after admission now revealed two hypodense areas in the region of both thalami (Figure 1).

She was discharged to a nursing home and when seen a year and 6 months later she was alert with normal attention span and orientated in all spheres. Formal mental tests (MMSE score 28/30) were normal. She had complete recovery of downward gaze and her gait was normal.

(C) The Fellowship of Postgraduate Medicine, 1989 


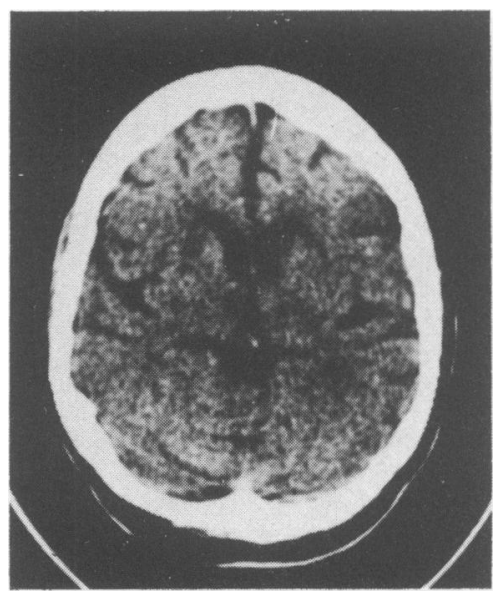

Figure 1 Bilateral thalamic hypodense areas consistent with infarction

\section{Discussion}

The clinical findings and symptomatology in our patient are consistent with a mesencephalothalamic involvement. The blood supply to the thalamus is complex and variable and this may be the reason why paramedian thalamic infarctions are usually accompanied by extensive infarction of the thalamus and midbrain.'

Vertical gaze palsy with somnolence and memory deficits has been described. ${ }^{1,4}$ However, isolated downward gaze paralysis is less common. ${ }^{5}$ In a study of five patients there was only one with the complete triad. ${ }^{6}$ The reason is that it can only be produced by two separate lesions one on each side involving the rostral interstitial nucleus of the medial longitudinal fasciculus and the nucleus of Darkschevitch as in our patient.

The stupor and subsequent hypersomnolence could be attributed to bilateral lesions in the intralaminar nuclei which are part of the rostral extension of the midbrain reticular activity system. ${ }^{7}$

The arterial supply to the thalamus has been divided into four territories-paramedian, posterior choroidal, infero-lateral and tuberothalamic-with the corres-

\section{References}

1. Meissner, I., Sapir, S., Kohmen, E. \& Stein, S.D. The paramedian diencephalic syndrome: a dynamic phenomenon. Stroke 1987, 18: 380-385.

2. Fisher, C.M. Lacunar strokes and infarcts. A review. Neurology (NY) 1982, 32: 871-876. ponding four clinical syndromes. ${ }^{8}$ As the vascular supply of the thalamus stems from different sources it seems likely that many nuclei are left functionally intact in thalamic lesions.

Damage to the medial dorsal nucleus of the thalamus produces an amnestic syndrome. ${ }^{9,10}$ Another thalamic structure that has been implicated with memory dysfunction is the anterior nucleus and the mammillothalamic tract. ${ }^{11}$

The misdiagnosis of amnestic patients as dementia may be more common than realized. In one study, $5 \%$ of the patients with the diagnosis of dementia were suffering from alcohol-related dysomnic syndrome. ${ }^{12}$ However, a 'subcortical' form of dementia with preservation of verbal IQ and related absence of aphasia, agnosia and verbal apraxia has been described with thalamic lesions. ${ }^{12}$

Our patient exhibited the classical triad. She had recovered from all of them when seen 18 months later. Meissner et al. ${ }^{\prime}$ described three patients and all of them showed bilateral thalamic infarcts on CT scan and the downward gaze deficiency remained relatively constant compared with the fluctuating course of the other symptoms. The impaired gaze improved or resolved in all of three. Most reports indicated poor prognosis and death within a year. ${ }^{13,14}$ The reason for this varied prognosis lies probably in the size of the lesions. Prolonged coma too seems to have a more dismal prognosis. Clinically the gait and such tasks as eating could be troublesome to the patient.

Lacunar syndromes are associated with deep infarcts or lacunes and result from occlusion of single perforating arteries by characteristic vascular lesions. This syndrome when it occurs is due to interruption of the vascular artery to the area from a single unpaired posterior thalamo-subthalmic artery ${ }^{15}$ and constitute a lacunar syndrome. Its occurrence following an ischaemic stroke indicates not only the site but also the responsible artery. ${ }^{16}$

The cause is embolic in $90 \%$ of cases. ${ }^{2}$ In our patient the source was probably cardiac, although cardioembolic sources are said to be less frequent among lacunar infarcts. ${ }^{17}$ The clinical deficit in patients with CT-documented basal ganglia infarcts due to cardiac embolism is more extensive than would be expected with lacunes and the CT shows a 'giant' or 'megalacune'. ${ }^{3}$
3. Weisberg, L.A. Editorial. Diagnostic classification of stroke especially lacunes. Stroke 1988, 19: 1071-1073. 
4. Swanson, R.A. \& Schmidley, J.W. Amnestic syndrome and vertical gaze palsy: early detection of bilateral thalamic infarction by CT and NMR. Stroke 1985, 16: $823-827$.

5. Cogan D. Paralysis of down-gaze. Arch Ophthalmol 1974, 97: 190-192.

6. Castigne, P., Lhermitte, F., Buge, A., Escourolle, R., Haan, J.J. \& Lyon-Caen, O. Paramedian thalamic and midbrain infarcts: clinical and neuropathological study. Ann Neurol 1981, 10: 127-148.

7. Guberman, A. \& Stuss, D. The syndrome of bilateral paramedian thalamic infarction. Neurology 1983, 33: $540-545$.

8. Bogousslavsky, J., Regli, F. \& Uske, A. Thalamic infarcts: clinical syndromes, etiology and prognosis. Neurology 1988, 38: 837-848.

9. Victor, M. The amnesic syndrome and its anatomic basis Can Med Assoc J 1969, 100: 1115-1125.

10. Speedie, L.J. \& Heilman, K.M. Amnestic disturbances following infarction of the left dorsomedial nucleus of the thalamus. Neuropsychologica 1982, 20: 597-604.
11. Hanky, G.J. \& Stewart-Wynne, E.G. Amnesia following thalamic haemorrhage another stroke syndrome. Stroke 1988, 19: 776-778.

12. Kiloh, J.J. \& Smith, L.G. the investigations of dementia: results of 100 consecutive cases of admissions. Lancet 1981, ii: $820-827$.

13. Jacobs, L. Anderson, P.J. \& Bender, M.B. The lesions producing paralysis of downward but not upward gaze. Arch Neurol 1973, 28: 319-323.

14. Halmagyi, G.H., Evans, W.A. \& Hallinan, J.M. Failure of downward gaze. Arch Neurol 1978, 35: 22-26.

15. Buttner-Ennrver, J.A. Buttner, U. Cohen, B. \& Baumgartner, G. Vertical gaze paralysis and the nostral interstitial nucleus of the medial longitudinal fasciculus. Brain 1982, 105: 125-149.

16. Kobari, M., Ishihara, N. \& Yunoki, K. Bilateral infarction associated with selective downward gaze paralysis. Eur Neurol 1987, 26: 246-251.

17. Bramford, J.M. \& Wailow, C.P. Evaluation and testing of the lacunar hypothesis. Stroke 1988, 19: 1074-1082. 